\title{
Quantum Mechanical Formulation of the Bloch NMR Flow Equations for the Analysis of Radio Wave Attenuation in Satellite Communication
}

\author{
Rasheedat Bola Abdulrahim ${ }^{1}$, Michael Dada ${ }^{2} \&$ Bamidele Awojoyogbe ${ }^{2}$ \\ ${ }^{1}$ National Space Research and Development Agency, Abuja, Nigeria \\ ${ }^{2}$ Department of Physics, Federal University of Technology, Minna, Niger State, Nigeria \\ Correspondence: Rasheedat Bola Abdulrahim, National Space Research and Development Agency, P.O. Box \\ 4086, Garki, Abuja, Nigeria. E-mail: bourlarnley@gmail.com
}

Received: December 10, 2012 Accepted: January10, 2013 Online Published: May 2, 2013

doi:10.5539/apr.v5n3p86

URL: http://dx.doi.org/10.5539/apr.v5n3p86

\begin{abstract}
Barrier potential in quantum mechanics is not just a theoretical phenomenon but is often encountered in various physical realities. It involves reflection and transmission of some wave functions around a region with a given potential function. It is possible for particles of a wave function to slow down or speed up if they manage to go through a potential barrier or a region, in which case, the observed change in their velocities is as good as saying that the particle's waves has gone through some processes of refraction. Therefore, reflection, transmission, absorption and refraction all involve phenomena which arise from the wave-like behavior of the motion of microscopic particles. Current analysis of the radio wave attenuation in satellite communications shows that there are no quantum mechanical models of the Bloch NMR flow equations for the analysis of radio wave attenuation in satellite communication system, especially in the area of the inter-dependence of the radio wave frequency and the refractive index of the atmosphere on the radio signal. This therefore necessitates the need to model and simulate the Bloch NMR flow equation quantum mechanically as presented in this study and use it for possible radio wave attenuation in satellite communication system for easy understanding by the earth-space link operators.
\end{abstract}

Keywords: quantum mechanics, Bloch NMR low equations, radio wave frequency, satellite communication

\section{Introduction}

It has been established that radio signals propagating between a spacecraft and ground experience various changes due to the presence of the ionosphere: Faraday rotation, propagation delay, refraction, dispersion, absorption and scintillation, with the first four of these directly dependent upon the columnar total electron content (TEC). Long-term radio-service planning requires quantification of the probabilities that user specified tolerable propagation effect levels are met (Robert, 2002). International Telecommunication union Recommendation (ITU-R) reliability expressions for HF are extended to Earth-space propagation. Formulae are quoted giving propagation degradation probabilities under multiple-effect conditions when these may be regarded as independent of one-another or when fully correlated. To a first order, Earth-space links are established by line-of-sight trajectories, with the various ionospheric effects representing a corruption of the free-space signals. The relative importance of these separate features and in relation to corresponding tropospheric factors which may often dominate will depend on frequency, geographical region and service application. For example, high-speed communication systems are principally affected by dispersion, absorption and scintillations, whereas for navigation and surveillance systems, propagation delay and refraction effects are likely to be of paramount concern. Often though, significant impairments may arise simultaneously from more than one of these phenomena (hawk.iszf.irk.ru, Jan 30, 2004)

\section{Theoretical Formulation}

The time-independent differential equation governing classical wave motion is of the same form as the time-independent Schrodinger equation (Awojoyegbe, 2007; Awojoyegbe, Faromika, M. Dada, \& E. Dada, 2011). 
For instance, electromagnetic radiation of frequency $f$, propagating through a medium with index of refraction $\mu$ obeys the equation:

$$
\frac{d^{2} \psi_{(x)}}{d x^{2}}+\left(\frac{2 \pi f \mu}{c}\right)^{2} \psi_{(x)}=0
$$

Where $\psi_{(\mathrm{x})}$ specifies the magnitude of the electric or magnetic field (Eiberg \& Resnick, 1985). Equation (1) has been compared with the time-independent Schrodinger equation and the quantum mechanical model of the Bloch NMR flow equation in the form (2):

$$
\frac{d^{2} \psi_{(x)}}{d x^{2}}+\frac{2 m}{\hbar^{2}}\left[E-V_{(x)}\right] \psi_{(x)}=0
$$

Equation (2) was derived based on the Bloch NMR flow equations with the index of refraction $\mu(\mathrm{x})$, accurately connected with the potential energy function by the relation (Awojoyegbe, 2007)

$$
\gamma B 1(x)=G(x)=\mu_{(x)}=\frac{c}{2 \pi f} \sqrt{\frac{2 m}{\hbar^{2}}\left(E-V_{(x)}\right)}
$$

where $\gamma$ is the gyromagnetic ratio, $\mathrm{B} 1(\mathrm{x})$ is the $\mathrm{rF}$ magnetic field, $\mathrm{E}$ is the total energy and $\mathrm{V}_{\mathrm{o}}(\mathrm{x})$ is the potential energy of the particles and $\mathrm{c}$ is the speed of light. Since Equation (1) holds for all electromagnetic radiations (Awojoyegbe, 2007; Eiberg \& Resnick 1985), then it must hold for all radio waves being transmitted from one point to the other including satellite communication). It is very important to also note that given the speed of transmission of radio waves, their dynamics can be said to be in steady state since variation of the magnitude of either the E-field or their B-field is not very much significant with time (Peter, 2003; Tri, 1990). Therefore Equation (1) is very much valid for radio wave transmission where $\psi_{(x)}$ can be taken to be the amplitude of the radio signal (either of the electric field or the magnetic field). If we therefore assume that $x$ is the distance (measured from the earth surface) as the radio wave travels from the ground station to the satellite and back, the variations in the magnitude of the radio wave along its path (trajectory) can be analyzed based on Equation (3). However, since particles of radio waves are electromagnetic particles which are mass less, we may need to re write Equation (3) based on simple quantum mechanics as

$$
\gamma B 1(x)=G(x)=\mu(x)=\frac{1}{h f}\{2 E(E-V(x))\}^{1 / 2}
$$

Equation (4) shows that the radio refractivity is essentially an energy phenomenon. That is it concerns the energy of the radio wave particles and the pulling of the atmospheric molecules.

The refractivity is the rate of change of $\mu$ with altitude (measured by $\mathrm{x}$ in this study). Changes in the rate is responsible for the curvature of radio rays. Information on the occurrence and types of gradients is required for radio path design and estimates of some propagation parameters .Gradients are either obtained from refractometers or computed from radiosonade data. From Equation (4),

$$
\frac{d \mu}{d x}=\frac{2 E f_{v}}{2 \hbar \omega \sqrt{2 E(E-V(x))}}
$$

Therefore, with data on $\frac{d \mu}{d x}$ obtained, we can always determine $f_{v}$ and $V(x)$ as these parameters are very crucial in determining the extent of radio attenuation expected from a given region of the atmosphere. It should be noted that all the losses (beam spreading loss, scintillation loss, weather loss, polarization loss, Rayleigh fading, free space loss, rain attenuation, and ionospheric losses. beam spreading loss, scintillation loss, weather loss, polarization loss, Rayleigh fading, free space loss, rain attenuation, and ionospheric losses) are all encompassed in $V(x)$ (Awojoyegbe, 2007; Eisberg \& Resnick, 1985).

\section{Analysis of Results}

Figure 1 shows the interaction of the refractive index of the transmitting medium and the distance from the earth station to the satellite in space. The software used to obtain the plot is mathematical tool. All the functions in the plots correspond to a particular atmospheric loss at any particular region and at a particular point in time. $\mathrm{n}$. The refractive index decreases as the distance from the origin increases. Also as the distance increases, the energy is reducing. The reduction in the energy of the propagated wave signifies that the signal has been attenuated.

Figure 1(f) shows that when $\mathrm{V}(\mathrm{x})=\cos \mathrm{kx}+\operatorname{sinkx}$, when $\mathrm{k}=1$, where $\mathrm{F}_{\mathrm{v}}$ varies with $\mathrm{x}$ as a trigonometric 
function. From the graph, the trigonometric function implies that for every $3 \mathrm{~km}$ (approx), the value of $\mathrm{F}_{\mathrm{v}}$ becomes zero thereafter it increases to a maximum/minimum of \pm 1.0 and this clearly an indication that at a particular distance, attenuation is high and at another point there is less attenuation. It is also observed that there is need to find a way of obtaining data on the refractivity gradient $\frac{d \mu}{d x}$ so that $f_{v}$ and $V(x)$ could be determined as these parameters are very crucial in determining the extent of radio attenuation expected from a given region of the atmosphere based on the value of constant $\mathrm{k}$, for a particular region

(a)
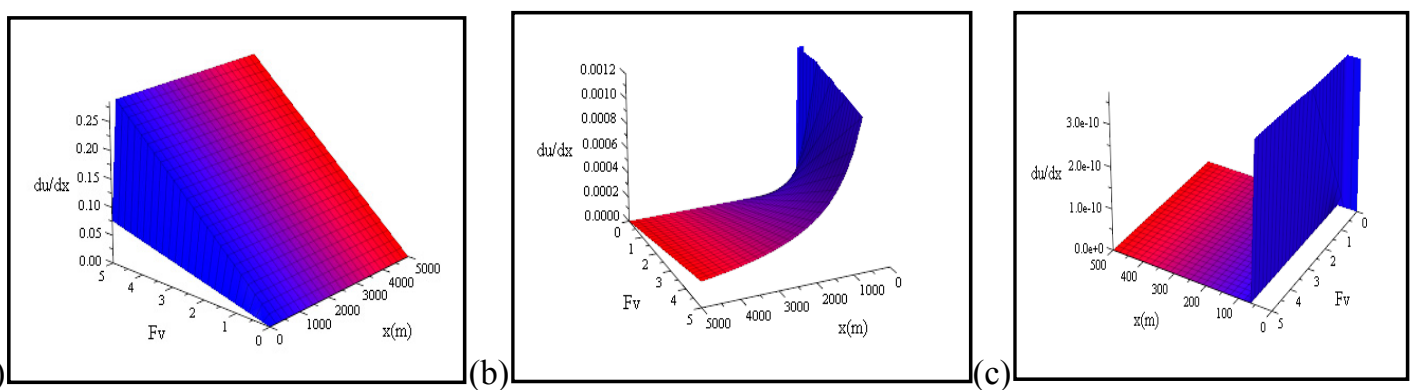

(d)
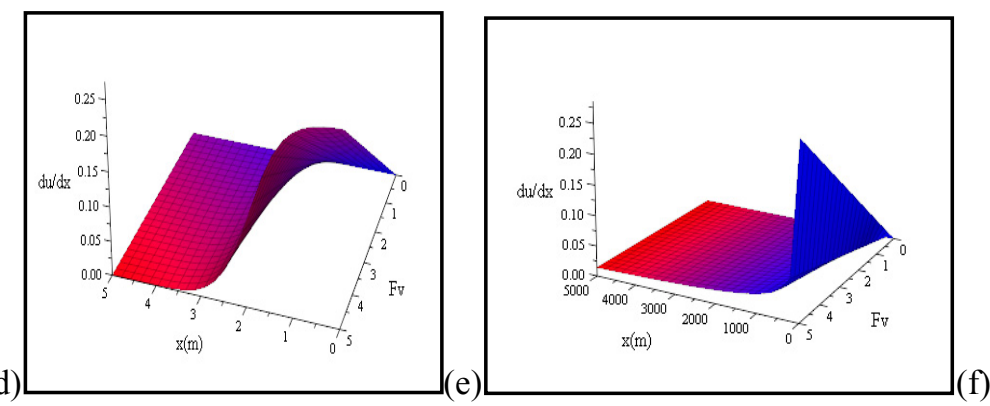

Figure 1. The Plot of refractivity gradient $\left(\frac{\mathrm{d} \mu(\mathrm{x})}{\mathrm{dx}}\right)$ against the atmospheric drag $\left(\mathrm{F}_{\mathrm{v}}\right)$ and the distance $(\mathrm{x})$ based on Equation (5) for (a) $\mathrm{V}(\mathrm{x})=\frac{\mathrm{k}}{\mathrm{x}}$, (b) $V(x)=k x^{2}$, (c) $\mathrm{V}(\mathrm{x})=-\mathrm{e}^{\mathrm{kx}}$, (d) $(\mathrm{x})=-\mathrm{e}^{\mathrm{kx}} \mathrm{x}^{2},(\mathrm{e}) \mathrm{V}(\mathrm{x})=\mathrm{kx}$, (f) $\mathrm{V}(\mathrm{x})=\cos \mathrm{kx}+\sin \mathrm{kx}$, when $\mathrm{k}=1$

\section{Conclusion}

In this study, we have shown that the modeled Bloch NMR flow equations for the analysis of radio wave attenuation in satellite communications can provide detailed description of the system quantum mechanically. The model can be an effective tool which may give a satellite operator the information necessary to launch a satellite into a particular region of the atmosphere at a particular point in the year. Therefore, once the behavior of the refractive index $\mu(\mathrm{x})$ is obtained from the weather information system, we could then manipulate $\mathrm{f}$, to minimize attenuation. A computational algorithm which describe in detail the dynamical state of the atmospheric particles applied to radio frequency waves as it travels from the ground station to the satellite and back, and the variations in the magnitude of the radio wave along its path (trajectory) using the Bloch NMR flow equations can thus be designed. The particles' properties of the modified time-independent Bloch NMR equations which describe the dynamics of atmospheric properties under the influence of $\mathrm{rF}$ magnetic field as presented here can stimulate further studies. The results obtained in Equation (4) clearly show the interdependence of the radio wave frequency and the refractive index of the atmosphere on the radio signal.

\section{References}

Awojoyegbe, O. B. (2007). A Quantum Mechanical Model of the Bloch NMR Flow Equations for Electron Dynamics in Fluids and the Molecular Level. Phys. Scr., 75, 788-794. http://dx.doi.org/10.1088/0031-8949/75/6/008

Awojoyogbe, O. B., Dada, M., Faromika, O. P., \& Dada. O. E. (2011). Mathematical concept of the Bloch flow equations for general magnetic resonance imaging: A review. Concepts in Magnetic Resonance Part A, 38A(3), 85-101. http://dx.doi.org/10.1002/cmr.a.20210 
Bradley, P. A. (2003). Ionospheric Propagation Degradation Probabilities For Earth-Space Links, Pandora, Scotland's Drive, Farnham Common, Slough, Berks, S12 3es, UK.

Eisberg, R., \& Resnick, R. (1985). Quantum Physics of Atoms, Molecules, Solids, Nuclei, and Particles (2nd ed., pp. 215-221). New York, NY: John Wiley \& Sons.

Ha, Tri. T. (1990). Digital Satellite Communications (2nd ed.). New York: McGraw Hill Publishing House.

Jones, R. W. (1985). Handbook on Satellite Communications (HSC, 3rd ed.). New York, NY: John Wiley \& Sons Inc.

Radicella, S. M., Nava, B., Coïsson, P., Kersley, L., \& Bailey, G. J. (2004). Effects of gradients of the electron densityon earth-space communications. Annals of Geophysics, Supplement, 47(2/3).

Wiley, J. L., \& James, R. W. (1996). Space Mission Analysis and Design (3rd ed.). El Segundo, Canada and Springer, New York, Space Technology Library: Microcosm Press. 